\title{
Correction to: Outcomes of a Behavioral Intervention for Sleep Disturbances in Children with Autism Spectrum Disorder
}

\author{
Sela Ann Sanberg ${ }^{1,2} \cdot$ Brett R. Kuhn $^{1} \cdot$ Abigail E. Kennedy $^{1}$
}

Published online: 16 August 2018

(c) Springer Science+Business Media, LLC, part of Springer Nature 2018

\section{Correction to: \\ Journal of Autism and Developmental Disorders https://doi.org/10.1007/s10803-018-3644-4}

The original version of this article unfortunately contained errors. The errors induced during the production process are corrected. The correct keywords, Figs. 1, 2, 3, 4, 5 and 6, and Tables 2, 4 are given below.

The Keywords should have appeared as: Keywords Autism - Sleep · Children · Parents · Behavioral intervention $\cdot$ Behavioral medicine

Author Contributions SS conceived of the study, participated in its design and coordination, participated in the measurement and data acquisition, performed the data analysis and interpretation, and drafted and revised the manuscript and figures; BK helped conceive the study, participated in its design, data analysis and interpretation, and manuscript revisions. AK participated in the coordination of the study, performed the measurement and data acquisition, and helped with manuscript revisions.

The original article can be found online at https://doi.org/10.1007/ s10803-018-3644-4.

Sela Ann Sanberg

sela.a.sanberg@wmich.edu

Brett R. Kuhn

brkuhn@unmc.edu

Abigail E. Kennedy

abigail.kennedy@unmc.edu

1 Department of Psychology, Munroe-Meyer Institute, University of Nebraska Medical Center (UNMC), 985450 Nebraska Medical Center, Omaha, NE 68198-5450, USA

2 Present Address: Department of Psychology, Western Michigan University, 1903 W. Michigan Ave., Kalamazoo, MI 49008-5439, USA 


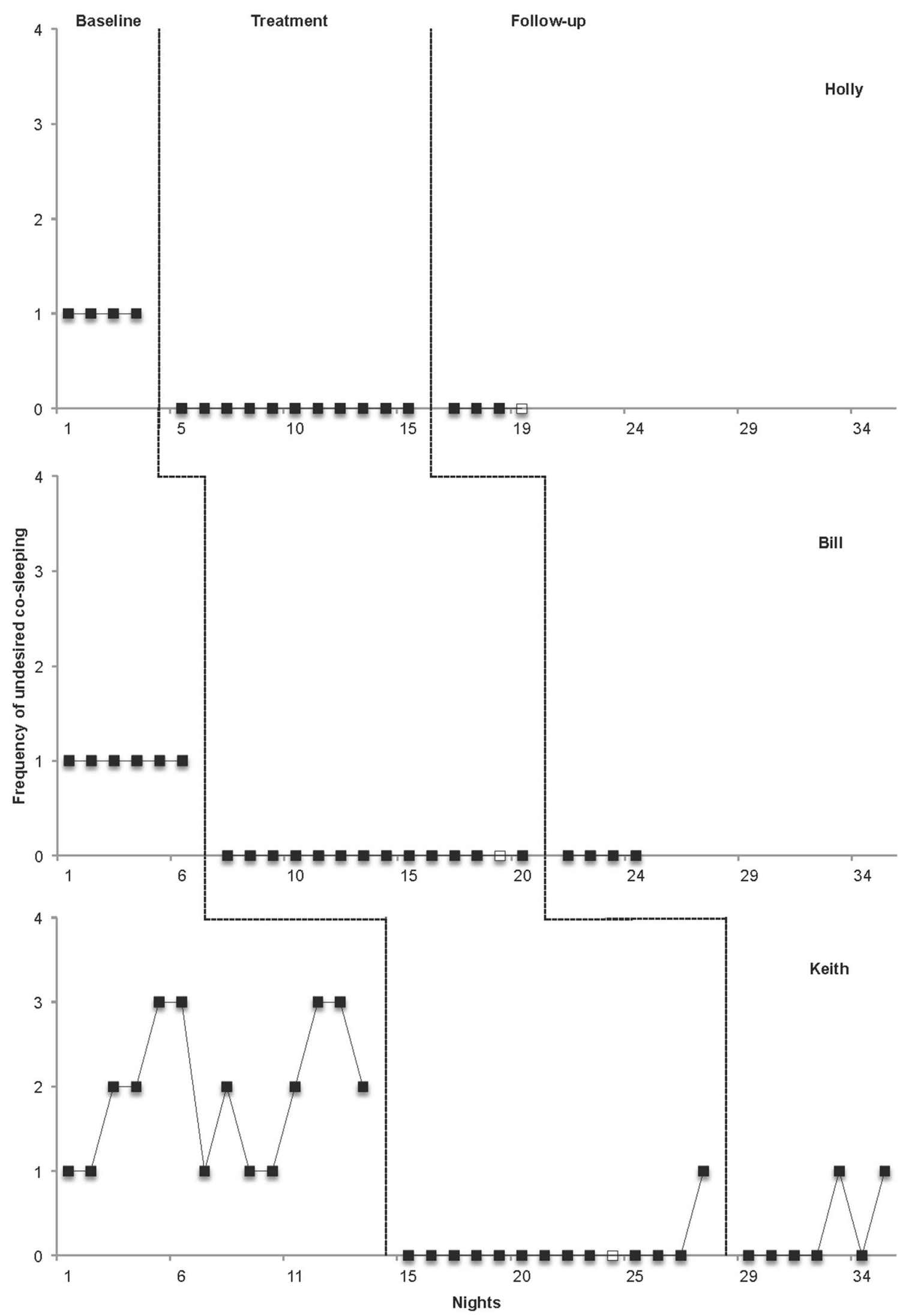

Fig. 1 Frequency of undesired co-sleeping per night with parents across children. Open squares represent a change in the child's routine 

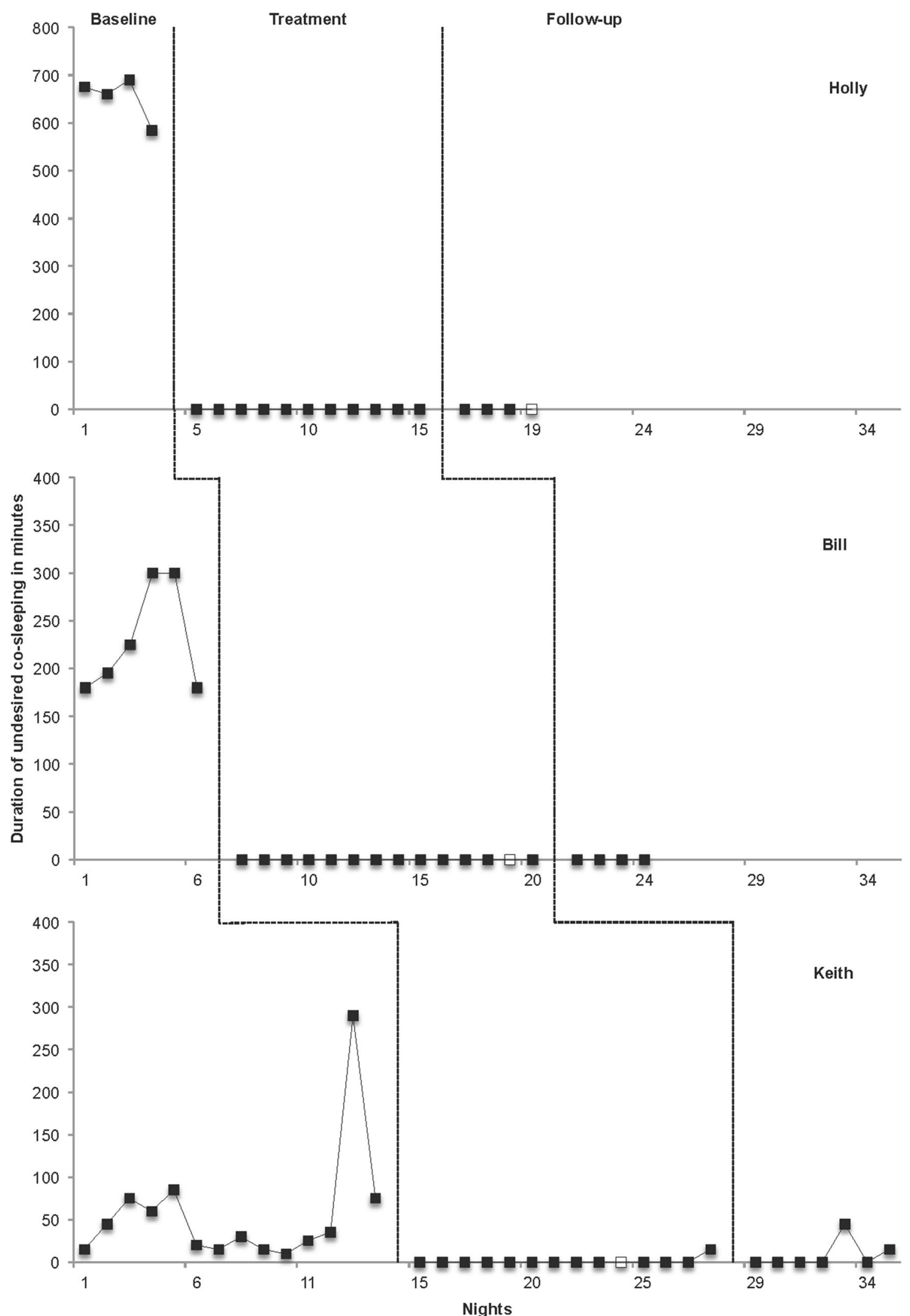

Fig. 2 Duration of undesired co-sleeping with parents in minutes (min) across children. Open squares represent a change in the child's routine 


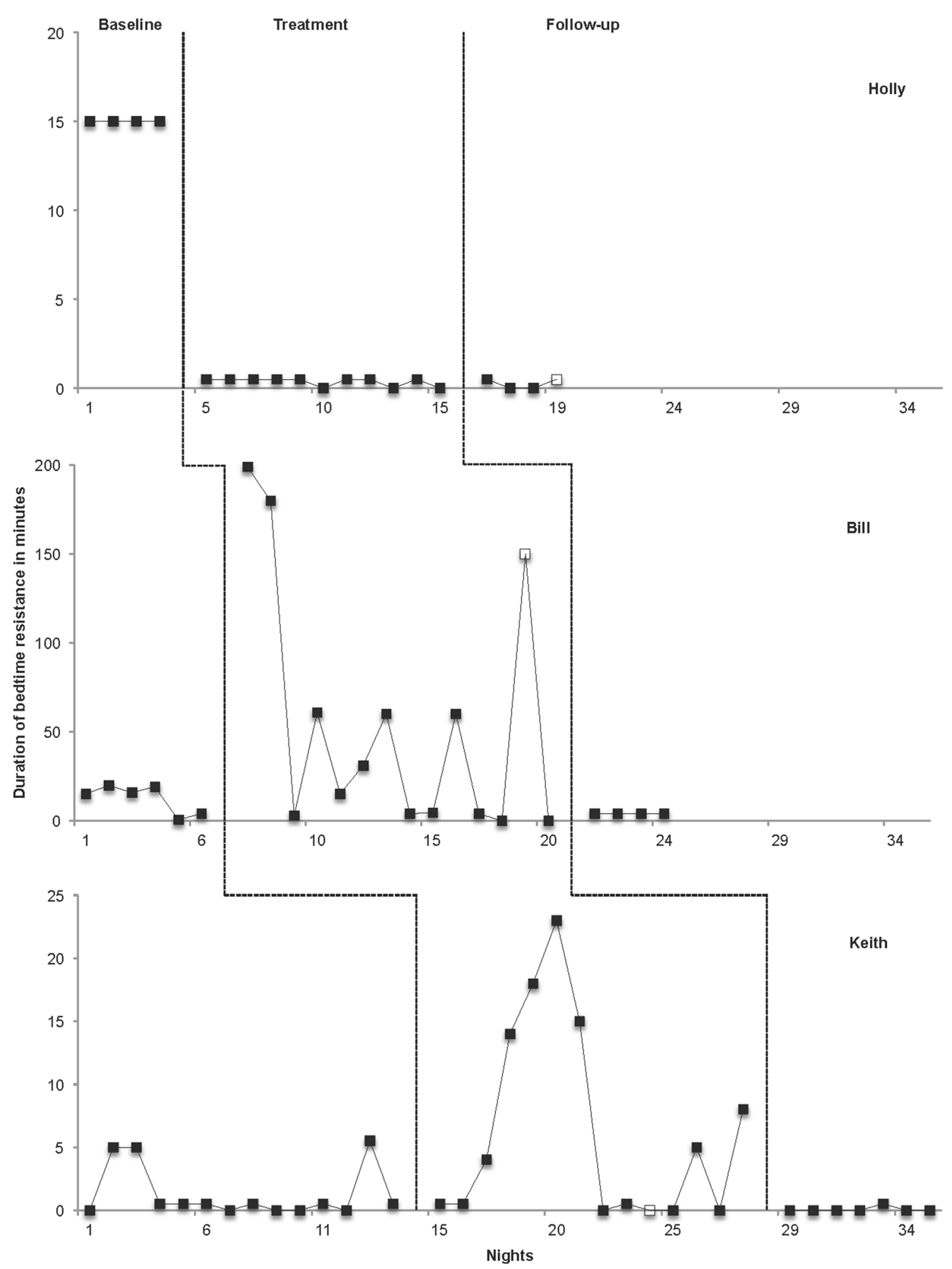

Fig. 3 Duration of bedtime resistance in minutes (min) across children. Open squares represent a change in the child's routine 


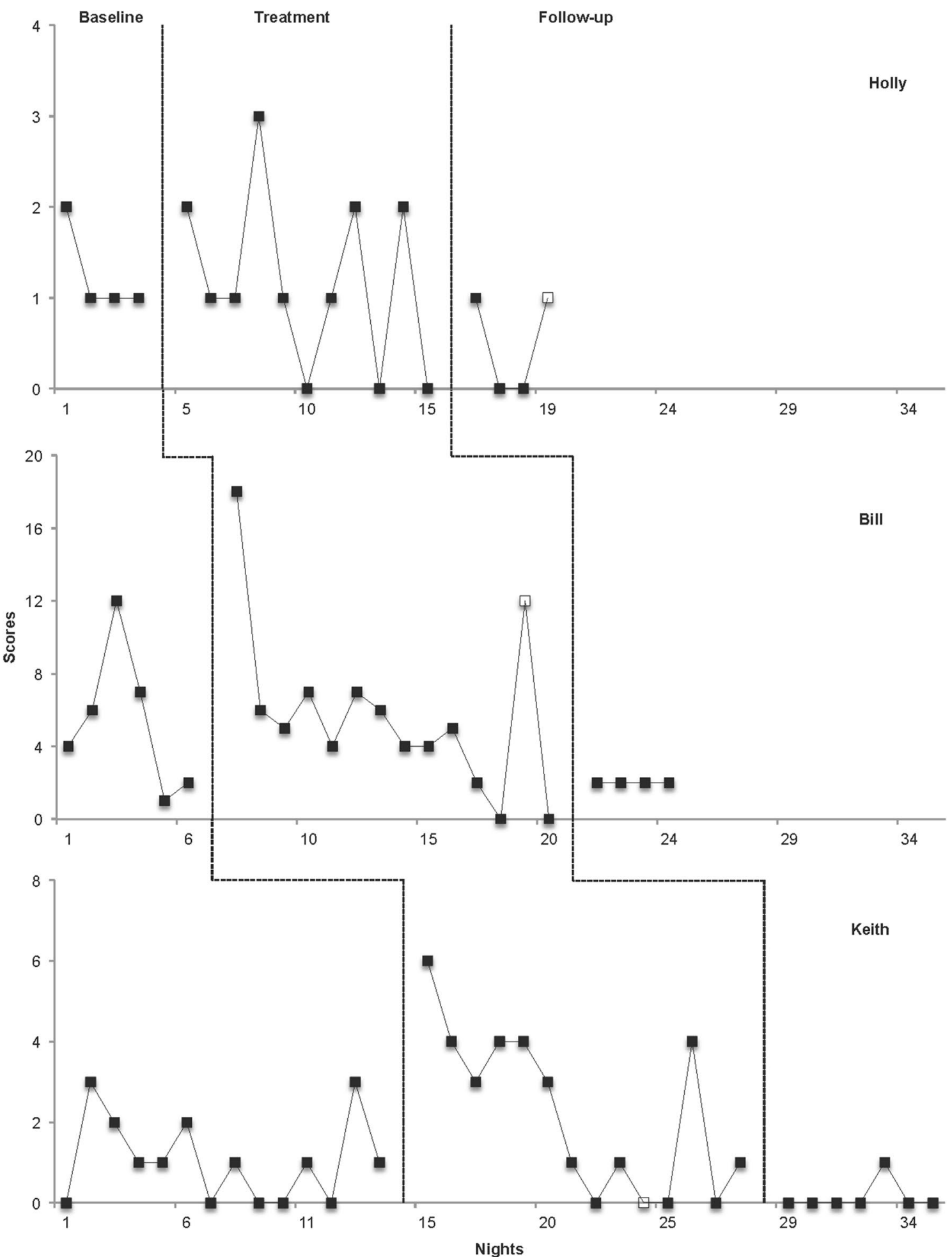

Fig. 4 Disruptive behavior composite scores across children. Open squares represent a change in the child's routine 


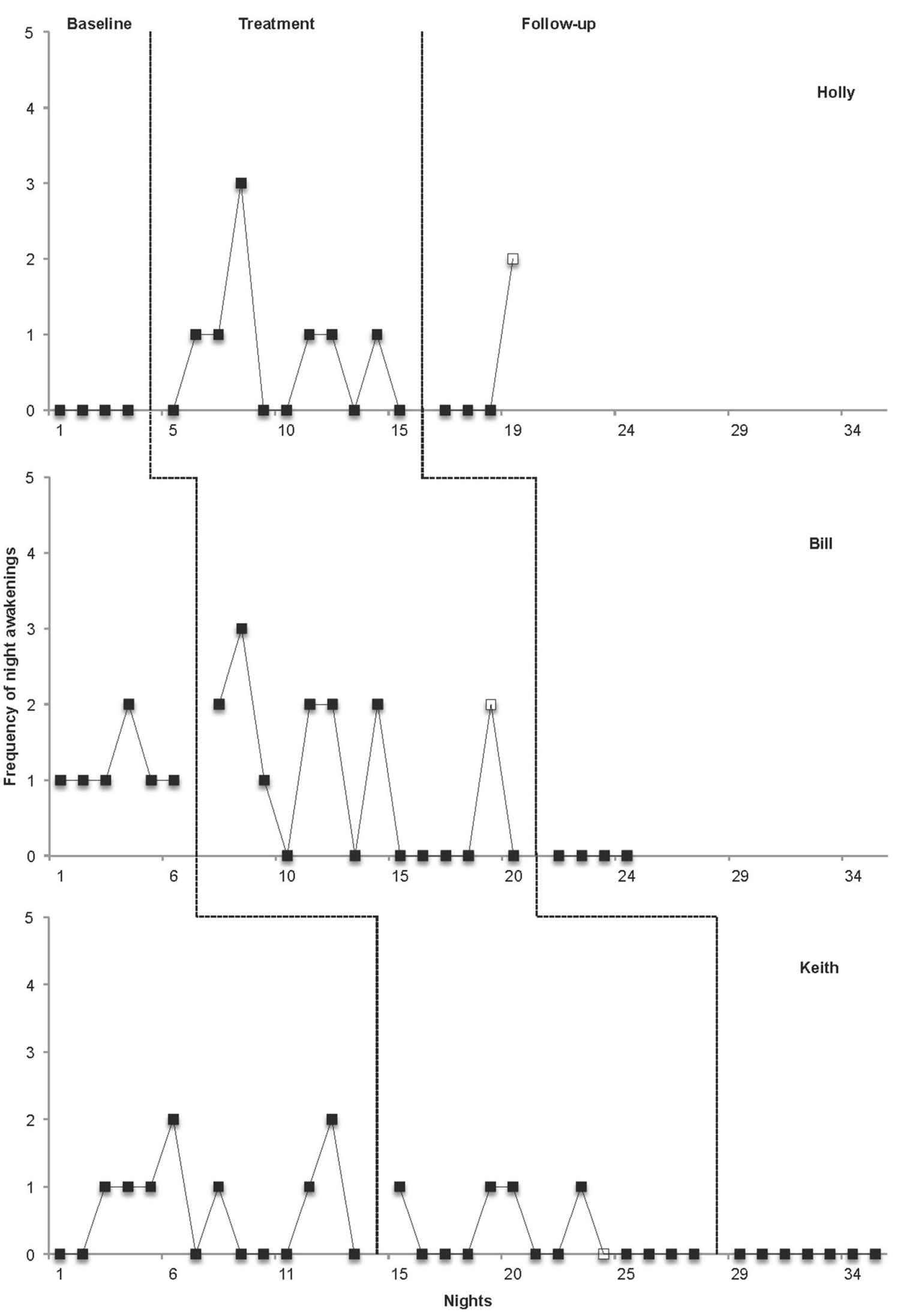

Fig. 5 Frequency of night awakenings across children. Open squares represent a change in the child's routine 


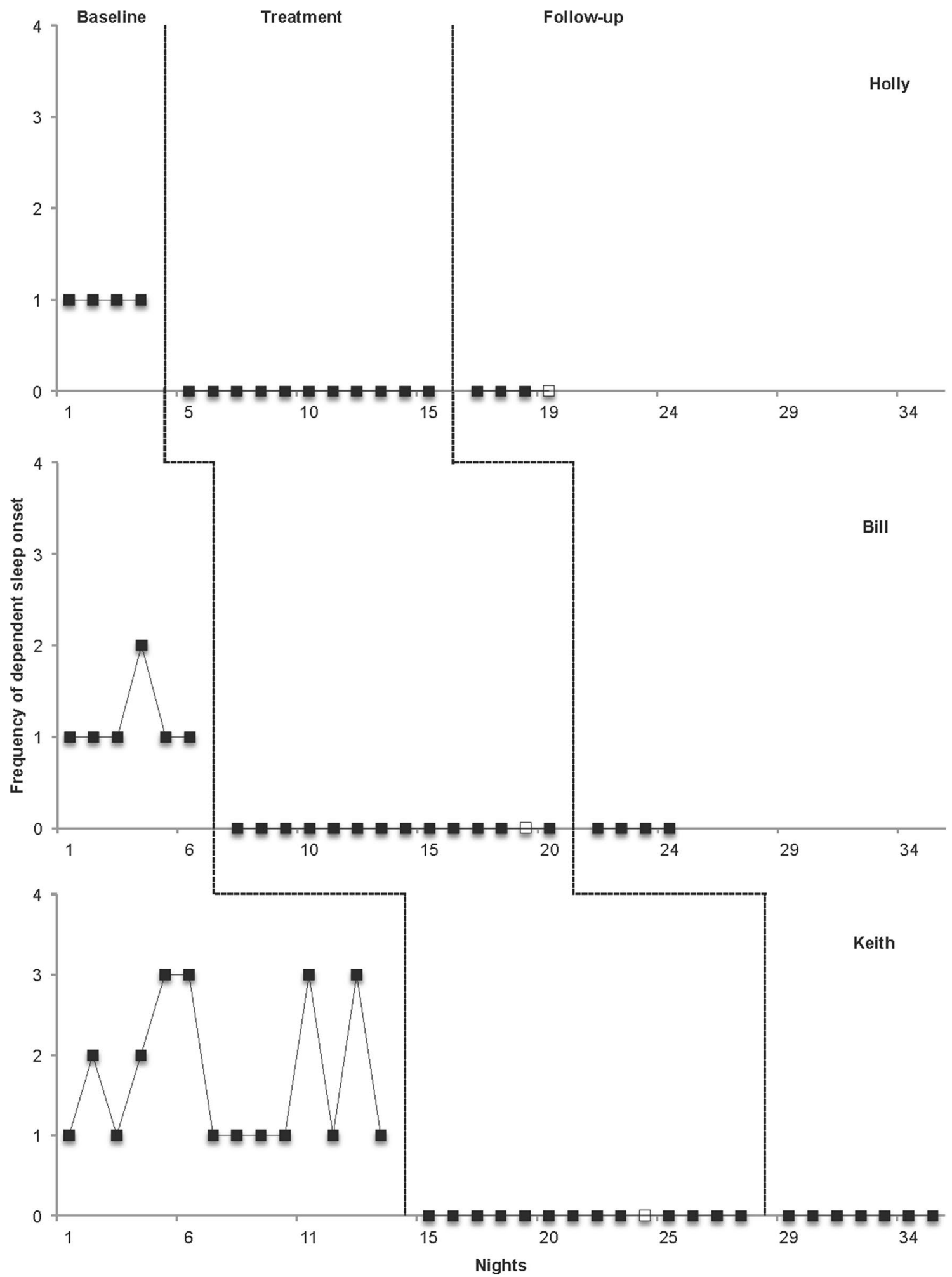

Fig. 6 Frequency of dependent sleep onset across children. Open squares represent a change in the child's routine 
Table 2 Dependent variables on sleep diary \& sleep related behavior diary

Dependent sleep variables Definitions

Frequency of night-awakenings (FNA)

Waking-up after sleep onset (WASO)

Sleep onset latency (SOL)

Total sleep time (TST)

Time in bed (TIB)

Dependent sleep onset (DSO)

Independent sleep onset (ISO)
Total number of times child awoke during the night and got out of bed; marked by time with an up arrow, not including final rising

Total minutes the child is awake after initially falling asleep; Non-shaded in boxes of time (small box $=15$ min.; large box $=1 \mathrm{~h}$ ) after initial sleep onset, until time of final rising

The time between initially being placed in bed awake, marked by time with a down arrow, and the time of sleep onset each night, marked by shaded in boxes

Total duration of nighttime and daytime sleep acquired within 24-h; Shaded in boxes for when the child is asleep (small box $=15 \mathrm{~min}$; large box $=1 \mathrm{~h}$ )

Amount of time the child was physically in bed during within 24-h; Time child gets into bed marked by a down arrow until final rising

The achievement of sleep with the required assistance of a parent; Marked by the letter D next to a down arrow

The achievement of sleep without the assistance of a parent; Marked by the letter I next to a down arrow

Dependent sleep related behavior variables

Definitions

Undesired co-sleeping (UC-S)

Unwanted co-sleeping with a parent or sibling in any room during a $24 \mathrm{~h}$ period; Frequency and duration of undesired co-sleeping with parent(s) or siblings by child

Sleeping in a non-designated area

Frequency of child sleeping in a location other than the child's bed (e.g., couch, car, floor, etc.) during a $24 \mathrm{~h}$ period

Location of undesired co-sleeping with child

Location of non-designated sleep 


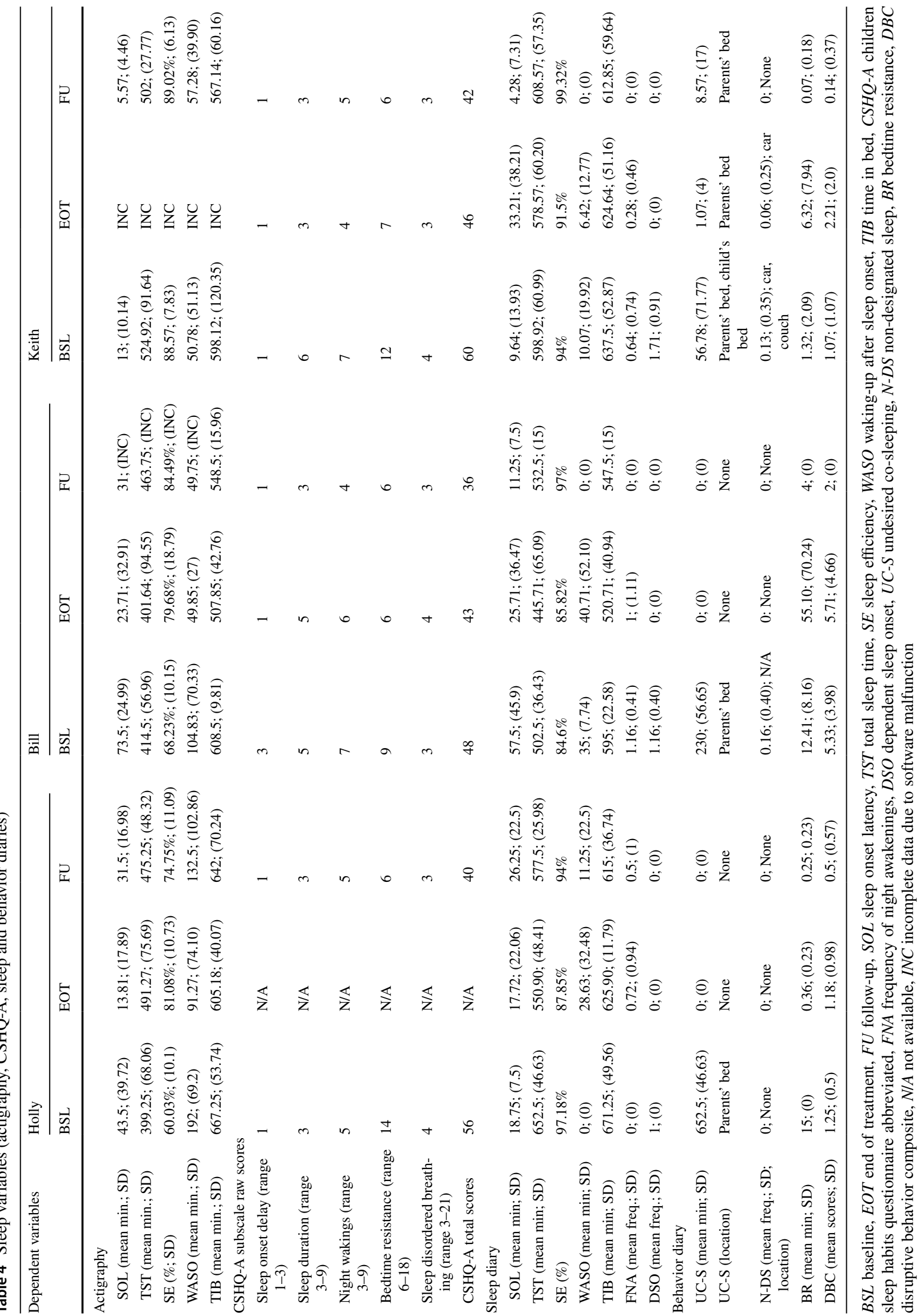

